\title{
Accelerated Vibrational Energy Relaxation of Water in Alkaline Environments
}

\author{
Roberto Cota, Sander Woutersen,* and Huib J. Bakker*
}

Cite This: J. Phys. Chem. B 2021, 125, 11980-11986

Read Online

ABSTRACT: We observe that hydrated hydroxide ions introduce an additional relaxation channel for the vibrational relaxation of the $\mathrm{OD}$ vibrations of $\mathrm{HDO}$ molecules in aqueous $\mathrm{NaOH}$ solutions. This additional relaxation path involves resonant (Förster) vibrational energy transfer from the excited $\mathrm{OD}$ vibration to $\mathrm{OH}$ stretch vibrations of hydrated $\mathrm{OH}^{-}$complexes. This energy transfer constitutes an efficient mechanism for dissipation of the OD vibrational energy, as the accepting $\mathrm{OH}$ stretch vibrations show an extremely rapid subsequent relaxation with a time constant of $<200$ fs. We find that the Förster energy transfer is characterized by a Förster radius of $2.8 \pm 0.2 \AA$.

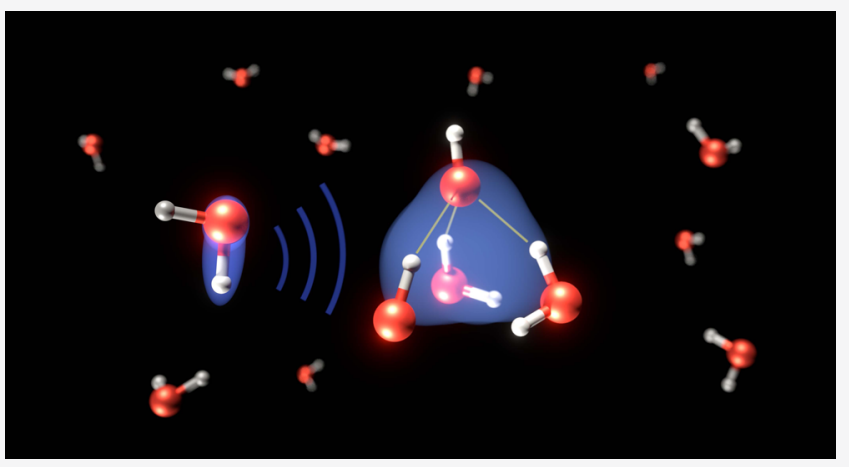

\section{INTRODUCTION}

Understanding the transfer of energy in water, the most ubiquitous liquid in nature, is of fundamental importance for many chemical and biological processes. In fact, the structural and dynamical properties of water facilitate a wide range of essential interactions in nature, such as protein folding. ${ }^{1}$ When energy is locally dissipated in an aqueous environment, the excess energy rapidly equilibrates due to structural rearrangements and intermolecular couplings. ${ }^{2-5}$ One of these couplings is dipole-dipole interaction leading to resonant (Förster) energy transfer. ${ }^{6}$ Vibrational Förster energy transfer has been observed for amide vibration of peptides, ${ }^{7}$ the $\mathrm{CO}$ vibration on metal surfaces, ${ }^{8,9}$ and the stretch vibrations of water and ice. ${ }^{2,5,10}$ In pure $\mathrm{H}_{2} \mathrm{O}$, Förster transfer between the $\mathrm{OH}$ stretch vibrations takes place on a time scale of $<100 \mathrm{fs}^{2}$

Förster energy transfer can also occur between vibrations of different molecular species. For instance, in acidic water, a Förster-type coupling between hydrated protons and OD vibrations has been observed to accelerate the relaxation of the latter vibrations. ${ }^{10}$ Here, we investigate whether a similar effect can occur in alkaline solutions. Recent theoretical studies have shown that hydroxide ions possess a different hydration structure compared to protons. ${ }^{11-16}$ We use ultrafast mid-IR pump-probe spectroscopy to investigate the vibrational relaxation dynamics of the OD stretch excitations of HDO molecules dissolved in aqueous solutions of hydroxide ions of different concentrations.

\section{EXPERIMENTAL METHODS}

Aqueous hydroxide solutions were prepared by diluting a commercially available solution of 50 wt $\% \mathrm{NaOH}$ (Sigma Aldrich) in deionized water $(5.5 \mu \mathrm{S} / \mathrm{m}, \mathrm{Milli}-\mathrm{Q})$ at concentrations ranging between 1 and $6 \mathrm{M}$. In addition, $\mathrm{D}_{2} \mathrm{O}(99.96 \% \mathrm{D}$, Eurisotop) was added to obtain an $\mathrm{OD} / \mathrm{OH}$ ratio of $4 \%$ in all studied samples. The samples were kept between two $2 \mathrm{~mm}$ thick $\mathrm{CaF}_{2}$ windows separated by Teflon spacers of $25 \mu \mathrm{m}$ for $1-3 \mathrm{M} \mathrm{NaOH}$ solutions and $10 \mu \mathrm{m}$ at higher concentrations. The path length did not affect the relaxation dynamics.

We perform polarization-resolved transient absorption measurements using femtosecond mid-infrared light pulses with a repetition rate of $1 \mathrm{kHz}$ and centered at a frequency of $2510 \mathrm{~cm}^{-1}$ that are resonant with the OD stretch vibration of HDO molecules in aqueous solution. These pulses are generated via parametric down-conversion of $\mathrm{Yb}$ :KGW laser pulses in a KTA crystal. At $2510 \mathrm{~cm}^{-1}$, the generated pulses have an energy of $12 \mu \mathrm{J}$, a bandwidth of $120 \mathrm{~cm}^{-1}$, and a pulse duration of $200 \mathrm{fs}$. At $3390 \mathrm{~cm}^{-1}$, the pulse energy is $25 \mu \mathrm{J}$, the bandwidth is $90 \mathrm{~cm}^{-1}$, and the pulse duration is $280 \mathrm{fs}$. A ZnSe window at an incident angle of $45^{\circ}$ is used to create the pump (transmission), while the reflected beam is sent over a second $\mathrm{ZnSe}$ window to generate the probe and reference pulses. The pump pulse is sent over a delay stage to vary the pump-probe time delay and sent through a $\lambda / 2$ plate to set its polarization at $\pi / 4$ with respect to the polarization of the probe. A rotating wire grid polarizer is used after the sample to selectively

Received: March 26, 2021

Revised: August 20, 2021

Published: October 21, 2021 
measure the polarization component of the probe that is parallel or perpendicular to the pump polarization.

We measure the pump-induced transient absorption changes $(\Delta \alpha)$ as a function of time delay between the pump and the probe. At $t=0$, the pump pulse excites OD stretches from the ground state to the first excited state, $|0\rangle \rightarrow|1\rangle$. This excitation leads to a reduced density of absorbers in the ground state and stimulated emission out of the excited state, both contributing to an absorption decrease (bleach). The excitation also leads to absorption at lower frequencies, associated with the $|1\rangle \rightarrow|2\rangle$ transition. The vibrational relaxation of the excited state eventually results in heating of the sample, which induces an absorption change that decays on a time scale (tens to hundreds of picoseconds) much slower than that of the pump-probe experiment.

The experimentally obtained parallel and perpendicular transient absorption signals are used to construct the isotropic transient response

$$
\Delta \alpha_{\text {iso }}(\omega, t)=\frac{1}{3}\left[\Delta \alpha_{\|}(\omega, t)+2 \Delta \alpha_{\perp}(\omega, t)\right]
$$

where $\omega$ and $t$ are the probe frequency and time delay, respectively. The isotropic signal provides information on the rate of vibrational energy relaxation and is insensitive to molecular reorientation.

\section{RESULTS}

Linear IR Absorption. Figure 1 displays the linear absorption spectra of solutions of $8 \% \mathrm{HDO}$ and different

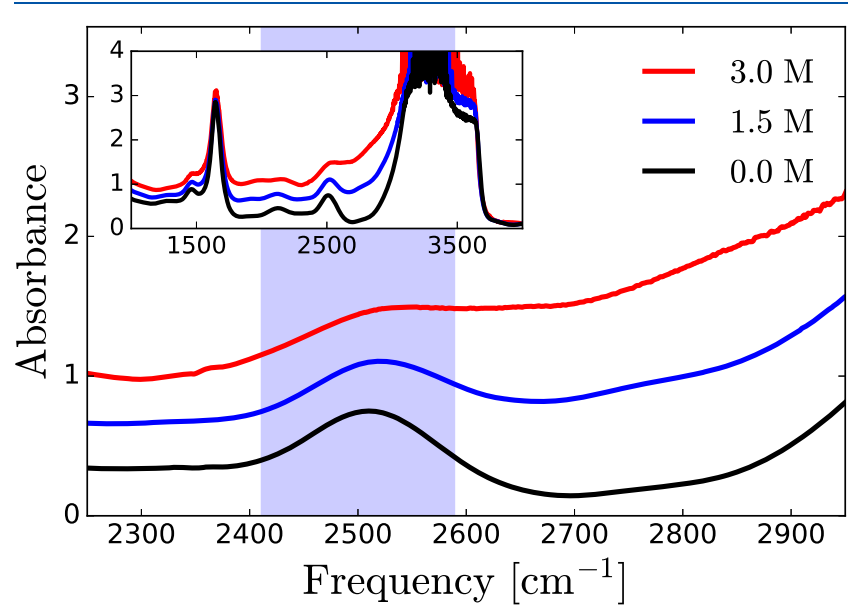

Figure 1. Linear IR spectra of $8 \% \mathrm{HDO}$ and different concentrations of $\mathrm{NaOH}$ in $\mathrm{H}_{2} \mathrm{O}$. The blue shaded area indicates the region where transient absorption is recorded.

concentrations of $\mathrm{NaOH}$ in $\mathrm{H}_{2} \mathrm{O}$. The spectra show a band centered at $2510 \mathrm{~cm}^{-1}$ that is assigned to the $\mathrm{OD}$ stretch vibration of $\mathrm{HDO}$ molecules that are hydrogen-bonded to neighboring water molecules. With increasing $\mathrm{OH}^{-}$concentration, a broad background absorption rises, which is attributed to the $\mathrm{OH}$ stretch vibrations of $\mathrm{H}_{2} \mathrm{O}$ molecules donating strong hydrogen bonds to $\mathrm{OH}^{-}$ions. The spectral responses of the $\mathrm{OD} / \mathrm{OH}$ stretch vibrations are negligibly affected by $\mathrm{Na}^{+}$ions.

Isotropic Transient Spectra. Figure 2 shows isotropic transient absorption spectra for neat $\mathrm{HDO} / \mathrm{H}_{2} \mathrm{O}$ and two $\mathrm{NaOH} / \mathrm{H}_{2} \mathrm{O}$ solutions. All solutions contain 8\% HDO: $\mathrm{D}_{2} \mathrm{O}$. The isotropic transient spectra show a pump-induced

\begin{tabular}{|c|c|c|c|c|c|c|c|c|}
\hline 0 & O & $0.4 \mathrm{ps}$ & $\square$ & $\square$ & $0.6 \mathrm{ps}$ & $\nabla$ & $\nabla$ & $1 \mathrm{ps}$ \\
\hline$\diamond$ & $\diamond$ & $2 \mathrm{ps}$ & $\Delta$ & $\Delta$ & $4 \mathrm{ps}$ & 0 & 0 & $50 \mathrm{ps}$ \\
\hline
\end{tabular}
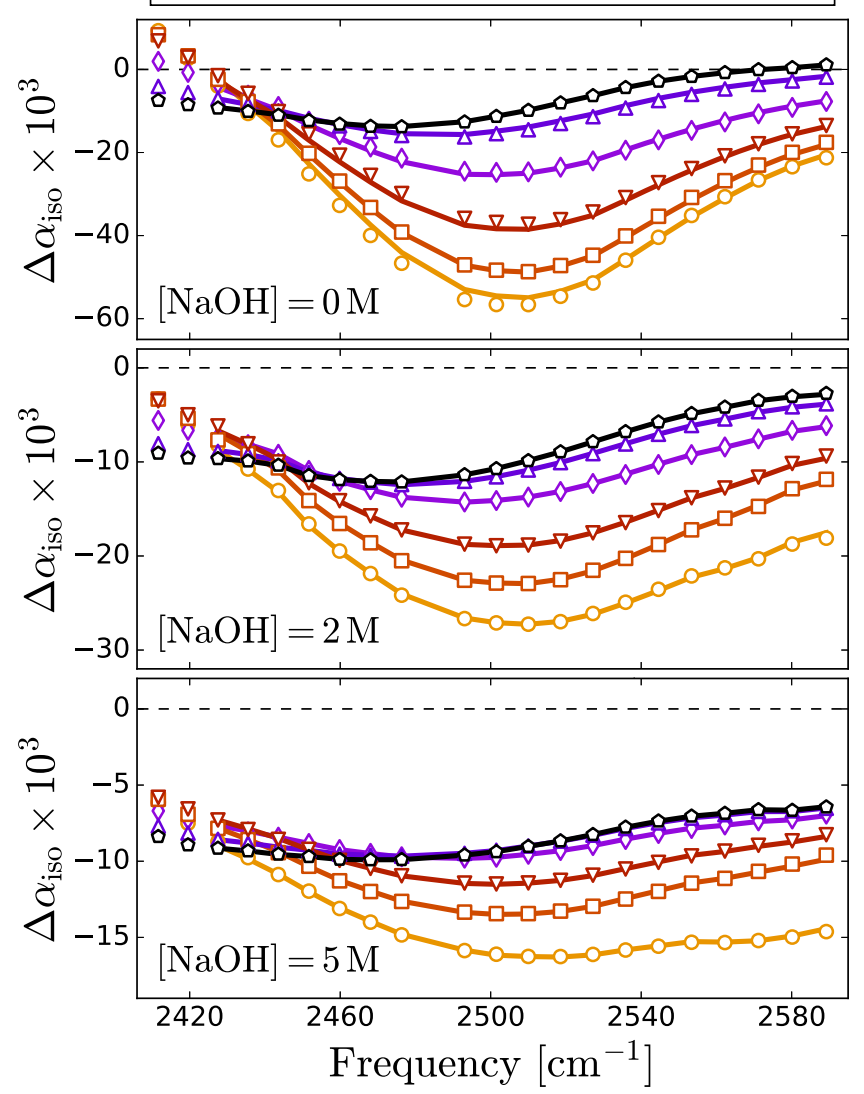

Figure 2. Isotropic transient absorption spectra for neat $\mathrm{HDO} / \mathrm{H}_{2} \mathrm{O}$ (upper panel) and $\mathrm{HDO}$ dissolved in $2 \mathrm{M}$ (middle panel) and $5 \mathrm{M}$ (lower panel) $\mathrm{NaOH} / \mathrm{H}_{2} \mathrm{O}$ solutions. The solid lines represent the results of a fit using the kinetic model described in the text.

bleaching signal at around $2510 \mathrm{~cm}^{-1}$ due to the excitation of the OD stretch vibration of the HDO molecules. At frequencies $<2420 \mathrm{~cm}^{-1}$, the upper panel also shows the highfrequency wing of the induced $|1\rangle \rightarrow|2\rangle$ absorption. The lower panels show that the amplitude of the bleaching signal of the OD stretch vibration decreases upon the addition of $\mathrm{NaOH}$ because an increasing part of the energy of the excitation pulse is absorbed by $\mathrm{OH}$ vibrations of $\mathrm{H}_{2} \mathrm{O}$ molecules that are hydrogen-bonded to $\mathrm{OH}^{-}$. In addition, with increasing $\mathrm{OH}^{-}$ concentration, the OD groups of an increasing fraction of the $\mathrm{HDO}$ molecules will be directly hydrogen-bonded to $\mathrm{OH}^{-}$, which shifts their absorption to lower frequency. As a result, these OD groups no longer contribute to $\sigma_{\mathrm{OD}}$, thus decreasing the amplitude of this spectral component in comparison to $\sigma_{\mathrm{OH}}$ and $\sigma_{\text {therm }}$.

To elucidate the transient spectral response of the excited $\mathrm{OH}$ stretch vibrations of $\mathrm{H}_{2} \mathrm{O}$ solvating $\mathrm{OH}^{-}$, we measure the transient absorption spectra of $\mathrm{NaOH} / \mathrm{H}_{2} \mathrm{O}$ solutions in a concentration range of $1-5 \mathrm{M}$ without the addition of $\mathrm{D}_{2} \mathrm{O}$ (see the Supporting Information). In these experiments, we only probe the vibrational relaxation dynamics of the ionassociated $\mathrm{OH}$ groups. The signal can be well described using a two-step relaxation model, in which the excited $\mathrm{OH}$ vibration relaxes rapidly within the solvation structure of the $\mathrm{OH}^{-}$ion, leading to a locally heated hydration complex with an associated intermediate-state spectrum $\sigma_{\mathrm{I}}$. This locally hot state equilibrates with the surroundings due to heat diffusion, 
thus creating a global hot ground state $\sigma_{\text {hgs }}$ This relaxation mechanism has been observed in a previous study on heat diffusion and molecular reorientation in aqueous hydroxide solutions. ${ }^{17}$

From a least-square fit of the isotropic data, we find that the two subsequent relaxation processes take place with time constants of $0.15 \pm 0.1$ and $0.5 \pm 0.1 \mathrm{ps}$, both independent of the concentration. The uncertainty in a time constant of 0.15 ps is relatively large as a result of the relatively long pulse duration of $0.2 \mathrm{ps}$. The spectral signature $\sigma_{\mathrm{OH}}$ of the excited state does not depend on concentration. The spectral shape of the intermediate state $\sigma_{\mathrm{I}}$ is very similar to that of the hot ground state $\sigma_{\text {hgs. }}$ The main difference is that $\sigma_{\mathrm{I}}$ has a much larger amplitude.

Spectral Decomposition and Population Dynamics. Based on these observations, we consider the transient absorption signal to be composed of the responses of two excited states and a thermal spectrum $\sigma_{\text {therm }}$. This latter spectrum $\sigma_{\text {therm }}$ represents both the spectral response $\sigma_{\mathrm{I}}$ of the intermediate state of the relaxation of the $\mathrm{OH}$ vibration of $\mathrm{H}_{2} \mathrm{O}$ molecules solvating $\mathrm{OH}^{-}$and the spectral response $\sigma_{\mathrm{hgs}}$ of the final hot ground state. We describe the responses of the intermediate state and the hot ground state with a single spectral component $\sigma_{\text {therm }}$ because these states have very similar spectral shapes. The time evolution of $\sigma_{\text {therm }}$ thus represents the population dynamics of both the intermediate state and the hot ground states. The transient absorption signal can thus be described as a linear combination of three states with associated transient spectra $\sigma_{j}$ and time-dependent populations $N_{j}$

$$
\Delta \alpha_{\text {iso }}=\sum_{j=1}^{3} N_{j}(t) \cdot \sigma_{j}(\omega)
$$

To determine the relaxation dynamics of the OD stretch vibration, we perform a spectral decomposition of the transient absorption spectra at all delay times and all concentrations. In this analysis, we fix the shape of the independently measured concentration-independent $\sigma_{\mathrm{OH}}$ band, and we fix the shape of the thermal spectrum $\sigma_{\text {therm }}$ at each studied $\mathrm{OH}^{-}$concentration to the transient absorption spectrum measured at long delay times ( $>30 \mathrm{ps}$ ). We treat the spectral shape of the excited OD vibration $\sigma_{\mathrm{OD}}$ as a global fit parameter across all $\mathrm{OH}^{-}$ concentrations. The global fit yields the spectral shape of $\sigma_{\mathrm{OD}}$ and the time-dependent population dynamics $N_{j}(t)$ of all three states without any assumption about the relaxation mechanism. The fitting procedure is described in detail in the Supporting Information. For a $2 \mathrm{M} \mathrm{NaOH} / \mathrm{H}_{2} \mathrm{O}$ solution, the $\chi^{2}$-minimization process yields the spectral components shown in Figure 3 and the population dynamics displayed in Figure 4. The solid red line in Figure 4 shows the dynamics of the thermal state, which represents the decay of the intermediate state of the relaxation of the $\mathrm{OH}$ vibration of $\mathrm{H}_{2} \mathrm{O}$ molecules solvating $\mathrm{OH}^{-}$at short delay times, and the ingrowing signal of the hot ground state at late delay times ( $>2 \mathrm{ps}$ ). The solid curves displayed in Figure 2 show that the spectral components shown in Figure 3 fit our experimental data well, even at the highest $\mathrm{OH}^{-}$concentration of $5 \mathrm{M}$.

In Figure 5, we show the normalized population dynamics of the excited OD vibration for five different $\mathrm{NaOH}$ concentrations. We observe that the relaxation becomes faster and increasingly nonexponential with increasing ion concentration. This ion-induced change of the relaxation can be explained by

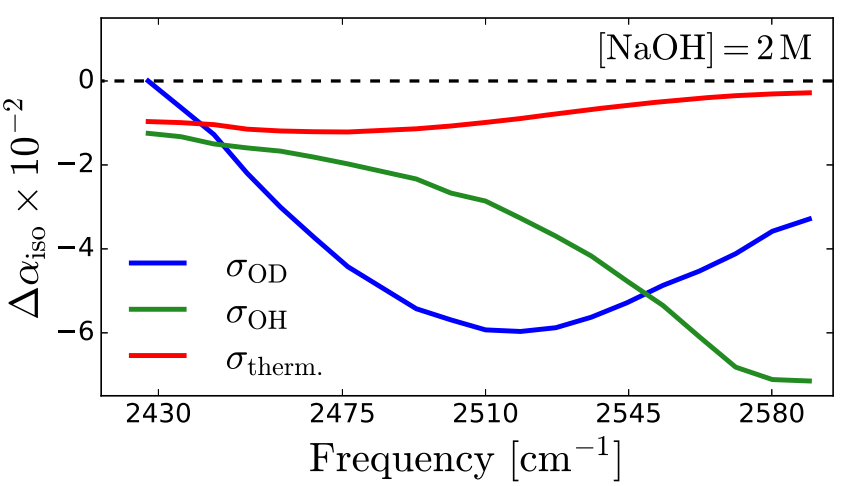

Figure 3. Spectral decomposition of the transient absorption spectrum of a solution of $\mathrm{HDO}$ and $2 \mathrm{M} \mathrm{NaOH}$ in $\mathrm{H}_{2} \mathrm{O}$. The spectra $\sigma_{\mathrm{OH}}$ and $\sigma_{\text {therm }}$ have been fixed in a least-squares optimization process of eq 2 to the data, while the spectral shape $\sigma_{\mathrm{OD}}$ of the OD vibrations is fitted as a global parameter across all $\mathrm{OH}^{-}$ concentrations.

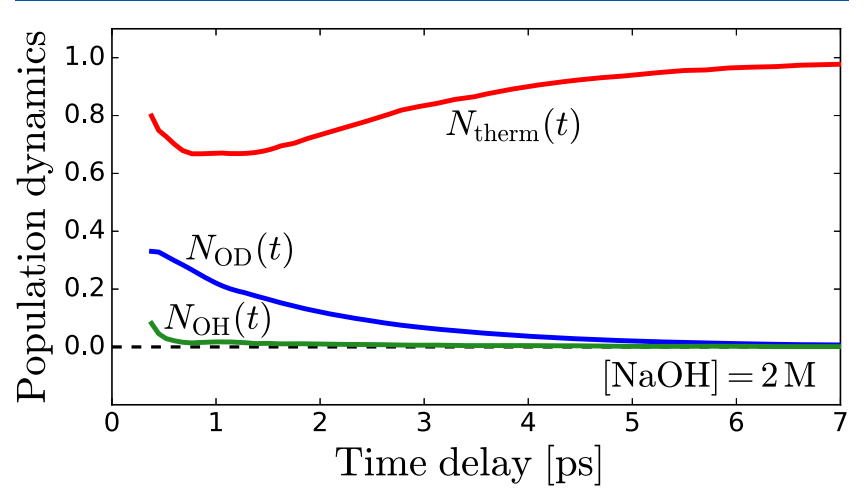

Figure 4. Fitted population dynamics of the different spectral components of Figure 3 of a solution of $\mathrm{HDO}$ and $2 \mathrm{M} \mathrm{NaOH}$ in $\mathrm{H}_{2} \mathrm{O}$. The population dynamics of the thermal spectrum represents both the decay of the intermediate state of the relaxation of the $\mathrm{OH}$ vibration of $\mathrm{H}_{2} \mathrm{O}$ molecules solvating $\mathrm{OH}^{-}$and the ingrowing signal of the hot ground state.

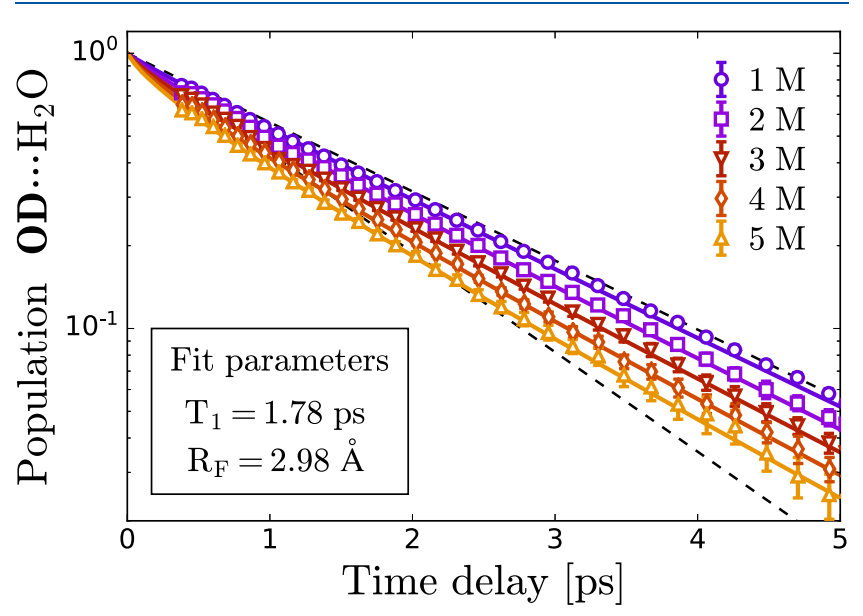

Figure 5. Time-evolving population probability of excited OD stretch vibrations at five different $\mathrm{NaOH}$ concentrations. The markers show the temporal decomposition described in the main text. The solid lines show the least-square fit of eq 5 , which results in $1.78 \mathrm{ps}$ for the OD vibrational lifetime and $2.98 \AA$ for the Förster radius. The dashed lines represent two single-exponential decays with time constants of 1.75 and 1.2 ps. The difference between the latter curve and the data illustrates that the observed decay is nonexponential. 
three possible scenarios: (i) the addition of $\mathrm{OH}^{-}$to the solution leads to inhomogeneous broadening of the OD absorption band with a faster relaxation rate at low OD frequencies than at high OD frequencies, thus yielding a nonexponential decay for $\sigma_{\mathrm{OD}}$; (ii) hydroxide ions affect the binding dynamics of the hydrogen-bond network, which in turn changes overall the vibrational relaxation process; (iii) the spectral overlap between the OD band and the hydroxide continuum enables resonant Förster energy transfer from excited OD vibrations to unexcited hydroxide complexes. To investigate scenario (i), we investigated the frequency dependence of the relaxation of the OD stretch vibration (see Figure S4 in the Supporting Information). We observe this relaxation to be uniform over the OD absorption band, thus ruling out the possibility that the nonexponential relaxation would originate from a frequency dependence of the OD relaxation rate.

To discriminate between scenarios (ii) and (iii) as origins of the ion-induced increase in the relaxation rate, we performed the same experiment on $\mathrm{H} / \mathrm{D}$-reversed isotope solutions, i.e., $\mathrm{H}_{2} \mathrm{O}$ diluted in $\mathrm{D}_{2} \mathrm{O}$. We thus measure the transient spectral response of solutions of $\mathrm{HDO}$ and $\mathrm{OD}^{-}$in $\mathrm{D}_{2} \mathrm{O}$, following the excitation of the $\mathrm{OH}$ stretch vibration of the HDO molecules near $3400 \mathrm{~cm}^{-1}$. Since the $\mathrm{OH}$ stretch vibration band does not overlap with the deuteroxide-induced continuum, as the inset in Figure 6 shows, the possibility of Förster energy transfer can be ruled out for these samples.

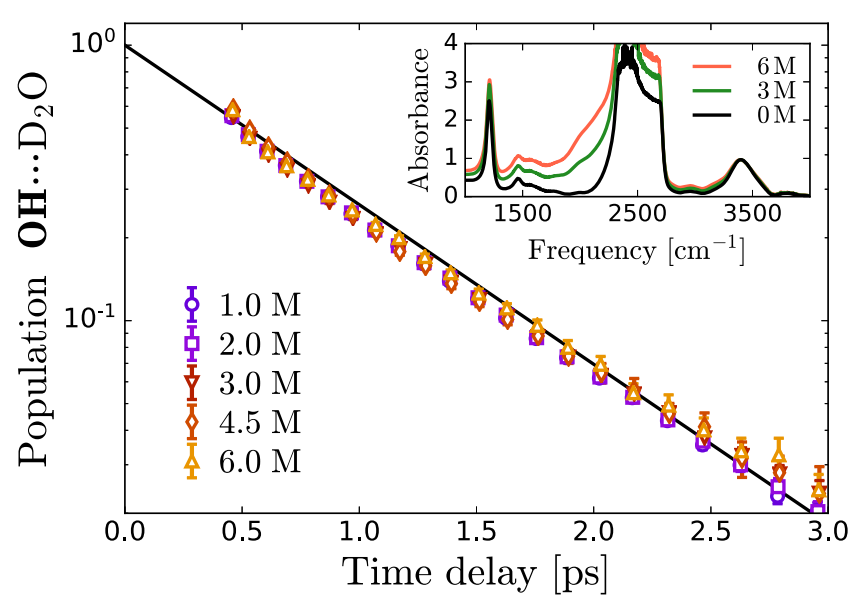

Figure 6. Time-evolving population probability of excited $\mathrm{OH}$ stretch vibrations at five different $\mathrm{NaOD}$ concentrations. The markers show the temporal decomposition described in the main text. The solid line represents a monoexponential decay with a time constant of $0.75 \mathrm{ps}$, similar to that in the neat $\mathrm{HDO} / \mathrm{D}_{2} \mathrm{O}$ solvent. ${ }^{19}$

We describe the data with a similar model that we used to describe the vibrational dynamics of the OD stretch vibration of $\mathrm{HDO}$ in $\mathrm{OH}^{-} / \mathrm{H}_{2} \mathrm{O}$ solutions and that we reported before. ${ }^{18}$ In this model, we consider two different types of excited $\mathrm{OH}$ stretch vibrations that relax via an intermediate state to a final hot ground state. The first type is formed by the $\mathrm{OH}$ vibration of HDO molecules that are not directly interacting with the $\mathrm{OD}^{-}$ions. The second type is formed by the $\mathrm{OH}$ vibrations of $\mathrm{HDO}$ molecules that are forming hydrogen bonds with $\mathrm{OD}^{-}$. These latter $\mathrm{OH}$ vibrations have a broad red-shifted spectral response centered at $3340 \mathrm{~cm}^{-1}$. Both types of $\mathrm{OH}$ vibrations relax to an intermediate state that subsequently relaxes to a hot ground state.
We find that for all studied $\mathrm{OD}^{-}$concentrations, the vibrationally excited $\mathrm{OH}$ groups that are directly hydrogenbonded to $\mathrm{OD}^{-}$ions relax with a time constant $T_{1}$ of $0.27 \pm$ $0.05 \mathrm{ps}$ and that the intermediate state relaxes with a time constant $0.9 \pm 0.3$ ps. The $\mathrm{OH}$ stretch vibrations of the $\mathrm{HDO}$ molecules that are not directly hydrogen-bonded to $\mathrm{OD}^{-}$ions relax with a time constant that shows negligible dependence on the $\mathrm{OD}^{-}$concentration, as illustrated in Figure 6. At all concentrations, the relaxation can be well described with an exponential function with a decay time constant of 0.75 ps. This relaxation time is similar to the previously reported time constant of 0.7 ps for the excited $\mathrm{OH}$ stretch vibration of $\mathrm{HDO}$ in neat $\mathrm{HDO} / \mathrm{D}_{2} \mathrm{O} .{ }^{19}$

In a previous study of the reorientation dynamics of the OD and $\mathrm{OH}$ vibrations of $\mathrm{HDO}$ molecules in aqueous hydroxide solutions, we did not explicitly include the vibrational relaxation of the $\mathrm{OH}$ groups that form hydrogen bonds with $\mathrm{OD}^{-}$, and we only considered the thermal effect following the relaxation of these vibrations. ${ }^{20}$ As a result, the observed relaxation rate represented the average relaxation dynamics of all $\mathrm{OH}$ vibrations, and this averaged relaxation becomes faster with increasing $\mathrm{OD}^{-}$concentration, as the contribution of $\mathrm{OH}$ groups directly bonded to $\mathrm{OD}^{-}$increases. In the present study, we investigate the $\mathrm{OH}$ stretch relaxation over a larger spectral range and we include the vibrational relaxation of the $\mathrm{OH}$ groups that form hydrogen bonds with $\mathrm{OD}^{-}$. We thus observe that for both the $\mathrm{OH}$ groups hydrating $\mathrm{OD}^{-}$and the $\mathrm{OH}$ groups that are not hydrating $\mathrm{OD}^{-}$, the vibrational relaxation rate shows negligible dependence on the $\mathrm{OD}^{-}$concentration. This means that there is no acceleration of the relaxation of the $\mathrm{OH}$ of $\mathrm{HDO}$ in $\mathrm{OD}^{-} / \mathrm{D}_{2} \mathrm{O}$ solutions with increasing $\mathrm{OD}^{-}$ concentration, only the relative contributions of the two $\mathrm{OH}$ species change.

It is interesting that for the $\mathrm{OH}$ vibration of $\mathrm{HDO}$ in $\mathrm{OD}^{-} /$ $\mathrm{D}_{2} \mathrm{O}$, we observe a fast relaxation component in the red wing of the $\mathrm{OH}$ absorption band, associated with $\mathrm{OH}$ groups that directly hydrate $\mathrm{OD}^{-}$ions, whereas for the $\mathrm{OD}$ vibration of $\mathrm{HDO}$ in $\mathrm{OH}^{-} / \mathrm{H}_{2} \mathrm{O}$ solutions, we do not see a similar component associated with OD groups directly hydrating $\mathrm{OH}^{-}$ (see Figure S4 in the Supporting Information). This observation suggests that these latter OD groups have a similar vibrational relaxation time constant as the OD groups that are further away from the $\mathrm{OH}^{-}$ions. A possible explanation for this difference between $\mathrm{OD}$ and $\mathrm{OH}$ vibrations is that the relaxation mechanisms differ, with the result that the relaxation of the $\mathrm{OH}$ vibration could be much more sensitive to the local hydrogen-bond strength than the relaxation of the OD vibration.

The absence of the concentration dependence of the relaxation of the $\mathrm{OH}$ vibrations of HDO molecules further away from $\mathrm{OH}^{-}$shows that the change of the hydrogen-bond network resulting from the addition of hydroxide ions has a negligible effect on the vibrational relaxation. Hence, the observed acceleration of the vibrational relaxation for the OD vibrations of $\mathrm{HDO}$ in $\mathrm{OH}^{-} / \mathrm{H}_{2} \mathrm{O}$ solutions can only be explained with the third scenario, i.e., Förster energy transfer from the excited $\mathrm{OD}$ vibration to $\mathrm{OH}$ vibrations of $\mathrm{H}_{2} \mathrm{O}$ molecules hydrating the $\mathrm{OH}^{-}$ions. This energy transfer is enabled by the spectral overlap of the OD stretch absorption spectrum and the wing of the broad and strong absorption band of the $\mathrm{OH}$ stretch vibrations of $\mathrm{H}_{2} \mathrm{O}$ molecules hydrating $\mathrm{OH}^{-}$ions. 
The observed uniform relaxation of the OD vibration at different frequencies (see Figure $S 4$ in the Supporting Information) is also consistent with a mechanism in which the nonexponential character of this relaxation is caused by Förster energy transfer, as the coupling with the very broad absorption of hydrated $\mathrm{OH}^{-}$complexes will not strongly depend on the OD frequency. In principle, the reverse Förster process from hydroxide complexes to $\mathrm{OD}$ vibrations can also occur. However, this interaction will have a negligible effect since vibrationally excited $\mathrm{OH}$ groups of $\mathrm{H}_{2} \mathrm{O}$ molecules hydrating $\mathrm{OH}^{-}$ions quickly relax with a time constant $<200 \mathrm{fs}$.

Förster Energy Transfer. The comparison between Figures 5 and 6 shows that Förster energy transfer to the hydrated $\mathrm{OH}^{-}$complexes serves as an effective additional relaxation channel for the excited OD vibrations. In the following, we will model the effect of this additional channel using the Förster formalism.

Within the Förster model, the rate of energy transfer between two dipoles is given by ${ }^{6}$

$$
k_{\mathrm{F}}(r)=\frac{1}{T_{1}}\left(\frac{R_{\mathrm{F}}}{r}\right)^{6}
$$

where $T_{1}$ is the intrinsic lifetime of the excited state and $r$ is the distance between the two interacting oscillators. The Förster radius $R_{\mathrm{F}}$ determines the distance at which $50 \%$ of the excitation energy is transferred via the Förster mechanism within the lifetime $T_{1}$ of the excitation. It thus follows that the Förster process becomes significant if the mean donoracceptor distance is comparable or smaller than $R_{\mathrm{F}}$, as will be the case at high $\mathrm{OH}^{-}$concentrations. Using eq 3 and assuming a radially uniform distribution of receiving oscillators, the survival probability of donors is given by ${ }^{6}$

$$
S(t, \rho)=\exp \left(-4 \pi \rho \int_{a_{0}}^{\infty} \mathrm{d} r r^{2}\left[1-e^{-k_{\mathrm{F}}(r) t}\right]\right)
$$

where $\rho$ is the density number of $\mathrm{OH}^{-}$ions and $a_{0}$ is the minimal approach distance, which is the excluded volume of a water molecule (in the limit of $a_{0}=0$, we recover the original formula reported by Förster). This expression can be used under the condition that the shapes of the absorption spectra of the donor and acceptor, i.e., of the OD vibration of HDO $\left(\sigma_{\mathrm{OD}}\right)$ and the $\mathrm{OH}$ vibrations of $\mathrm{H}_{2} \mathrm{O}$ hydrating $\mathrm{OH}^{-}\left(\sigma_{\mathrm{OH}}\right)$, do not significantly change when the concentration of $\mathrm{OH}^{-}$ increases. Indeed, we find strong evidence that the shapes of these spectra hardly change. For $\sigma_{\mathrm{OH}}$, this can be seen in the inset of Figure 1 and in Figure $S 1$ in the Supporting Information where we show the difference absorption spectra obtained by subtracting the absorption spectrum of $\mathrm{H}_{2} \mathrm{O} / \mathrm{D}_{2} \mathrm{O}$ from the spectra of $\mathrm{OH}^{-} / \mathrm{H}_{2} \mathrm{O} / \mathrm{D}_{2} \mathrm{O}$ for different $\mathrm{OH}^{-}$ concentrations. The inset of Figure 1 and the normalized difference spectra of Figure S1 show that the shape of the lowfrequency tail of the absorption spectrum of $\mathrm{H}_{2} \mathrm{O}$ molecules hydrating $\mathrm{OH}^{-}$is independent of the concentration of $\mathrm{OH}^{-}$. For the OD vibration of $\mathrm{HDO}\left(\sigma_{\mathrm{OD}}\right)$, we find that we can accurately model the transient absorption spectra using the same shape of $\sigma_{\mathrm{OD}}$ at all studied $\mathrm{OH}^{-}$concentrations. This latter observation also implies that the dipole-dipole (Förster) coupling of the $\mathrm{OD}$ vibrations and the $\mathrm{OH}$ vibrations does not lead to strong mixing of these vibrations and that the perturbative Förster approach remains valid even at high $\mathrm{OH}^{-}$concentrations.
Using the Förster model to describe the data implies that we assume that all of the acceptors have the same absorption spectrum and that the acceptors are statistically distributed. The absorption spectrum of the $\mathrm{OH}$ stretch vibrations of $\mathrm{H}_{2} \mathrm{O}$ will be inhomogeneously broadened, meaning that different acceptors have different spectra. However, it is not a priori clear whether the species for which the spectra better overlap with the OD band of HDO are closer or further away from the energy donating the OD stretch vibration of HDO, so these effects may well average out. Probably the strongest approximation in using the Förster expression for energy transfer in aqueous environments is that the distribution of distances and relative angles of donors and acceptors is assumed to be statistical. In view of the hydrogen-bond structure of water and hydroxide solutions, this assumption is not valid for short donor-acceptor distances. Hence, the Förster model is expected to break down at high concentrations where short donor-acceptor distances are important.

Assuming that the intrinsic vibrational relaxation process and the Förster energy transfer are independent of each other, the time dependence of the excited population is given by

$$
N(t, \rho)=\exp \left(-\frac{t}{T_{1}}-4 \pi \rho \int_{a_{0}}^{\infty} \mathrm{d} r r^{2}\left[1-e^{-k_{\mathrm{F}}(r) t}\right]\right)
$$

We fit this expression to the experimental data shown in Figure 5 with $T_{1}$ and $R_{\mathrm{F}}$ as global parameters. We fix $a_{0}$ to the previously reported value of $1.5 \AA$ for OD vibrations in aqueous solutions. ${ }^{10}$ The resulting fits are represented by the solid curves in Figure 5. The intrinsic vibrational lifetime $T_{1}$ of the OD vibration obtained from the fit is $1.78 \pm 0.05 \mathrm{ps}$, which agrees well with the results of previous studies. ${ }^{5,21}$ We find a Förster radius of $2.8 \pm 0.2 \AA$, which is similar to the characteristic intermolecular distance of liquid water. ${ }^{22,23}$

\section{DISCUSSION}

We find that the hydration complexes of $\mathrm{OH}^{-}$can act as antennae receiving energy from nearby excited vibrations. Because of their broad, nearly continuous absorption spectrum, we can expect that hydrated hydroxide ions can quench the energy of any IR-active vibration between 1000 and 3400 $\mathrm{cm}^{-1}$, like amide and carbonyl vibrations $\left(\sim 1700 \mathrm{~cm}^{-1}\right)$ that are ubiquitous in (bio)chemistry. As such, hydrated hydroxide ions can act as effective dissipators of excess vibrational energy in aqueous chemical reactions.

Our findings are in line with previous observations for protonated aqueous solutions. ${ }^{10}$ The hydrated proton shows a similar continuum absorption band as the hydrated hydroxide ion $^{24}$ and is also observed to accept energy from excited OD vibrations, thereby accelerating their relaxation. ${ }^{10}$ For the coupling between OD vibrations and hydrated protons, a larger Förster radius of $\sim 4 \AA$ was found, showing that hydrated protons are even more efficient acceptors of vibrational energy than hydrated hydroxide ions. This difference in the efficiency of Förster energy transfer between acidic and basic solutions is consistent with the stronger absorption of low-frequency $\mathrm{OH}$ stretch vibrations in acidic solutions compared to the absorption of these vibrations in basic solutions, at corresponding $\mathrm{H}^{+} / \mathrm{OH}^{-}$concentrations. This difference in absorption strength indicates that the number density and/or transition dipole of low-frequency $\mathrm{OH}$ species is higher in acidic solutions than in basic solutions, thus providing more 
(efficient) acceptors of the energy of the OD stretch vibrational excitation of the HDO molecules.

\section{CONCLUSIONS}

We studied the vibrational relaxation mechanism of OD stretch excitations of $\mathrm{HDO}$ molecules in $\mathrm{NaOH} / \mathrm{H}_{2} \mathrm{O}$ solutions using femtosecond mid-infrared transient absorption spectroscopy. We decomposed the measured transient spectra into spectral components corresponding to the excited OD stretch vibration of the $\mathrm{HDO}$ molecules, the excited $\mathrm{OH}$ stretch vibrations of $\mathrm{H}_{2} \mathrm{O}$ molecules hydrating $\mathrm{OH}^{-}$ions, and the hot ground state that results after the vibrational relaxation is completed. This analysis yielded the population dynamics of all three states. With increasing concentration of $\mathrm{OH}^{-}$ions in solution, we found the decay of the excited OD vibration to become faster and to deviate increasingly from a single-exponential decay. These observations indicate the presence of an additional relaxation channel for the OD vibrations. This additional path is assigned to Förster resonance energy transfer from OD stretches to the $\mathrm{OH}$ stretch vibrations of hydrated $\mathrm{OH}^{-}$ complexes. From a global analysis of the relaxation dynamics at different $\mathrm{OH}^{-}$concentrations, we determined the intrinsic vibrational lifetime of the $\mathrm{OD}$ stretch vibration to be $1.78 \pm$ $0.05 \mathrm{ps}$, and the Förster radius to be $2.8 \pm 0.2 \AA$. Our results show that hydrated $\mathrm{OH}^{-}$complexes can serve as effective antennae and dissipators of excess vibrational energy in aqueous chemical reactions.

\section{ASSOCIATED CONTENT}

\section{SI Supporting Information}

The Supporting Information is available free of charge at https://pubs.acs.org/doi/10.1021/acs.jpcb.1c02730.

Linear absorption spectra; transient absorption signals of solutions of $\mathrm{NaOH}$ in $\mathrm{H}_{2} \mathrm{O}$; detailed description of the fitting procedure (PDF)

\section{AUTHOR INFORMATION}

\section{Corresponding Authors}

Sander Woutersen - Van 't Hoff Institute for Molecular Sciences, University of Amsterdam, 1098 XH Amsterdam, Netherlands; @ orcid.org/0000-0003-4661-7738; Email: s.woutersen@uva.nl

Huib J. Bakker - AMOLF, 1098 XG Amsterdam, Netherlands; 10 orcid.org/0000-0003-1564-5314; Email: h.bakker@amolf.nl

\section{Author}

Roberto Cota - Van 't Hoff Institute for Molecular Sciences, University of Amsterdam, 1098 XH Amsterdam, Netherlands; AMOLF, 1098 XG Amsterdam, Netherlands; (ㄱ) orcid.org/0000-0002-5270-6158

Complete contact information is available at:

https://pubs.acs.org/10.1021/acs.jpcb.1c02730

\section{Notes}

The authors declare no competing financial interest.

\section{ACKNOWLEDGMENTS}

The authors kindly thank Hinco Schoenmaker for technical support, and Eliane van Dam for fruitful discussions. This work is part of the research program of the Netherlands
Organization for Scientific Research (NWO), grant number 12PR2989.

\section{REFERENCES}

(1) Bellissent-Funel, M.-C.; Hassanali, A.; Havenith, M.; Henchman, R.; Pohl, P.; Sterpone, F.; van der Spoel, D.; Xu, Y.; Garcia, A. E. Water determines the structure and dynamics of proteins. Chem. Rev. 2016, 116, 7673-7697.

(2) Woutersen, S.; Bakker, H. J. Resonant intermolecular transfer of vibrational energy in liquid water. Nature 1999, 402, 507-509.

(3) Deàk, J. C.; Rhea, S. T.; Iwaki, L. K.; Dlott, D. D. Vibrational energy relaxation and spectral diffusion in water and deuterated water. J. Phys. Chem. A 2000, 104, 4866-4875.

(4) Elsaesser, T. Ultrafast memory loss and relaxation processes in hydrogen-bonded systems. Biol. Chem. 2009, 390, 1125-1132.

(5) Piatkowski, L.; Eisenthal, K. B.; Bakker, H. J. Ultrafast intermolecular energy transfer in heavy water. Phys. Chem. Chem. Phys. 2009, 11, 9033-9038.

(6) Förster, Th. Zwischenmolekulare Energiewanderung und Fluoreszenz. Ann. Phys. 1948, 437, 55-75.

(7) Fang, C.; Senes, A.; Cristian, L.; DeGrado, W. F.; Hochstrasser, R. M. Amide vibrations are delocalized across the hydrophobic interface of a transmembrane helix dimer. Proc. Natl. Acad. Sci. U.S.A. 2006, 103, 16740-16745.

(8) Bonn, M.; Hess, C.; Wolf, M. The dynamics of vibrational excitations on surfaces: $\mathrm{CO}$ on $\mathrm{Ru}(001)$. J. Chem. Phys. 2001, 115, $7725-7735$

(9) Roeterdink, W. G.; Berg, O.; Bonn, M. Frequency-and timedomain femtosecond vibrational sum frequency generation from $\mathrm{CO}$ adsorbed on $\mathrm{Pt}(111)$. J. Chem. Phys. 2004, 121, 10174-10180.

(10) Timmer, R. L. A.; Tielrooij, K. J.; Bakker, H. J. Vibrational Förster transfer to hydrated protons. J. Chem. Phys. 2010, 132, No. 194504.

(11) Tuckerman, M. E.; Marx, D.; Parrinello, M. The nature and transport mechanism of hydrated hydroxide ions in aqueous solution. Nature 2002, 417, 925-929.

(12) Chen, B.; Ivanov, I.; Park, J. M.; Parrinello, M.; Klein, M. L. Solvation Structure and Mobility Mechanism of $\mathrm{OH}^{-}$: A CarParrinello Molecular Dynamics Investigation of Alkaline Solutions. J. Phys. Chem. B 2002, 106, 12006-12016.

(13) Hassanali, A.; Prakash, M. K.; Eshet, H.; Parrinello, M. On the recombination of hydronium and hydroxide ions in water. Proc. Natl. Acad. Sci. U.S.A. 2011, 108, 20410-20415.

(14) Chen, M.; Zheng, L.; Santra, B.; Ko, H. Y.; Distasio, R. A.; Klein, M. L.; Car, R.; Wu, X. Hydroxide diffuses slower than hydronium in water because its solvated structure inhibits correlated proton transfer. Nat. Chem. 2018, 10, 413-419.

(15) Drexler, C. I.; Miller, T. C.; Rogers, B. A.; Li, Y. C.; Daly, C. A.; Yang, T.; Corcelli, S. A.; Cremer, P. S. Counter Cations Affect Transport in Aqueous Hydroxide Solutions with Ion Specificity. J. Am. Chem. Soc. 2019, 141, 6930-6936.

(16) de Oliveira, D. M.; Bredt, A. J.; Miller, T. C.; Corcelli, S. A.; Ben-Amotz, D. Spectroscopic and Structural Characterization of Water-Shared Ion-Pairs in Aqueous Sodium and Lithium Hydroxide. J. Phys. Chem. B 2021, 125, 1439-1446.

(17) Liu, L.; Hunger, J.; Bakker, H. J. Energy Relaxation Dynamics of the Hydration Complex of Hydroxide. J. Phys. Chem. A 2011, 115, 14593-14598.

(18) Cota, R.; van Dam, E. P.; Woutersen, S.; Bakker, H. J. Slowing Down of the Molecular Reorientation of Water in Concentrated Alkaline Solutions. J. Phys. Chem. B 2020, 124, 8309-8316.

(19) Rezus, Y. L. A.; Bakker, H. J. Orientational dynamics of isotopically diluted $\mathrm{H}_{2} \mathrm{O}$ and $\mathrm{D}_{2} \mathrm{O}$. J. Chem. Phys. 2006, 125, No. 144512.

(20) Hunger, J.; Liu, L.; Tielrooij, K.-J.; Bonn, M.; Bakker, H. Vibrational and orientational dynamics of water in aqueous hydroxide solutions. J. Chem. Phys. 2011, 135, No. 124517.

(21) Rezus, Y. L. A.; Bakker, H. J. On the orientational relaxation of HDO in liquid water. J. Chem. Phys. 2005, 123, No. 114502. 
(22) Perkins, S. J. Protein volumes and hydration effects: The calculations of partial specific volumes, neutron scattering matchpoints and 280-nm absorption coefficients for proteins and glycoproteins from amino acid sequences. Eur. J. Biochem. 1986, 157, 169-180.

(23) Chen, X.; Weber, I.; Harrison, R. W. Hydration water and bulk water in proteins have distinct properties in radial distributions calculated from 105 atomic resolution crystal structures. J. Phys. Chem. B 2008, 112, 12073-12080.

(24) Thämer, M.; De Marco, L.; Ramasesha, K.; Mandal, A.; Tokmakoff, A. Ultrafast 2D IR spectroscopy of the excess proton in liquid water. Science 2015, 350, 78-82. 